\title{
EDITORIAL
}

\section{The mycobacterial mystery}

\author{
N. Schönfeld
}

B efore the advent of HIV, which led to an increased awareness of Mycobacterium avium complex disease, followed by the first prospective comparison studies on treatment regimens $[1,2]$, the topic of nontuberculous mycobacterial disease was a true example of Aristotelian science. Previously, only a few experts worldwide had compiled empirical data and disseminated knowledge on the subject, which was based upon meticulous observations in small series or case studies from the 1950s onwards [3]. These studies demonstrated the manifold particularities of M. avium complex disease and others as compared with tuberculosis, and also showed how even nontuberculous mycobacterial lung diseases alone, quite apart from manifestations in other organs, appear in widely varying forms. To this day, an ever increasing number of reports on uncommon or recently detected and potentially pathogenic species with strange names deepen the impression that we are dealing with an intricate matter.

In 1979, WOLINSKY [4] published his legendary review on nontuberculous mycobacteria (NTM) and associated diseases, which is still worth reading and citing: "The pathogenesis of adult pulmonary disease is obscure. Does it represent primary infection after inhalation of aerosolized infected droplet nuclei that then localize and proliferate in specific areas of damaged lung? Or is it a reactivation of dormant bacilli that had been acquired previously?" [4]. Meanwhile, the history of HIV especially, and the growing number of patients with transplants, have helped in understanding the role, and some mechanisms, of systemic immunosuppression as an important factor in the development of both disseminated and pulmonary disease. Have we, however, deepened our understanding of the pathophysiology of localised lung disease in non-HIV patients, this being a key issue?

Focusing on treatment, in 1985 another pioneer in the field of tuberculosis and nontuberculous mycobacterial diseases, Karl Ludwig Radenbach (1918-1986), wrote: "Clinically, treatment cannot rely on controlled trials, due to the small number of cases. However, retrospective analyses carried out by experienced pneumologists demonstrate that if individualised multiple drug combinations are adjusted to the susceptibility test results of each strain, this helps to avoid secondary bacterial resistance and leads to satisfactory treatment outcome" [5]. The substantial variations in drug susceptibility between species and between different strains within the same species alone prompted RADENBACH [5] to state that no standard

CORRESPONDENCE: N. Schönfeld, Lungenklinik Heckeshorn, HELIOS Klinikum Emil von Behring, Zum Heckeshorn 33, 14109 Berlin, Germany. Fax: 49 3080022778. E-mail: Schoenfeld.Berlin@ T-online.de therapy exists. RADENBACH [5] also drew attention to another fact that other authors frequently leave unmentioned: "The basis of chemotherapy is the intensive treatment of resistancereducing underlying disease." Prospective trials followed and diagnostic and therapeutic recommendations, even guidelines, were elaborated [6, 7]. However, was it wise to broadly reject an individualised therapeutic approach in favour of regimens that are only specific to certain species, and to disregard the vast polymorphism of ubiquitous mycobacteria and the widely varying ways in which they affect the human body?

The prospective nationwide study presented by DailLoux et al. [8] in the current issue of the European Respiratory Journal (ERJ) reports on epidemiological data, clinical findings and treatment results in no fewer than 263 patients. Estimating the incidence at 0.73 per 100,000, DAILloux et al. [8] have documented the persistent presence of a disease group that is responsible for substantial morbidity and a consistently reported mortality. In general, the demographic transformation in Western societies, with rising percentages of older people and a corresponding increase in predisposing diseases, may in turn lead to increasing numbers of patients in the future, although this trend is not yet clearly discernible. With regard to patient characteristics, radiological patterns, the spectrum of underlying diseases and the regional differences in the incidence of species, the study group failed to find any results that improve our understanding of this rare disease complex. The summary of the treatment results confirms the variable and, in some cases, unsatisfactory data published by other working groups.

In the tradition of thorough clinical observation aimed at expanding our knowledge of mycobacterial disease, in the current issue of the ERJ, FOWLER et al. [9] report on the significant incidence of mycobacterial disease or colonisation (synonymous with infection without evidence of pulmonary disease) in bronchiectasis patients. The central finding seems to be that it was not possible to identify clinical subgroups of patients, or other relevant influencing factors, which might be associated with a higher probability of cultures positive for NTM. With two out of nine patients in the culture-positive group versus two out of 75 in the culture-negative group bearing a cystic fibrosis (CF) transmembrane conductance regulator mutation, it is hardly correct to say that due to $\mathrm{CF}$ bronchiectasis patients have a higher risk of being affected by NTM. A more important finding is that patients with positive cultures for Pseudomonas aeruginosa are not less likely to develop mycobacterial disease. On the contrary, the opposite could be true. The association between positive mycobacterial cultures and the finding of mucous plugging in computed 
tomography may just be an indication of a poor bronchial clearance, which could predispose to either infectious complication.

The astonishingly frequent ancillary finding of more than one species in cultures from the same patient in the study by FOWLER et al. [9] brings us to the almost notorious ongoing battle between clinicians and microbiologists as to how to interpret positive-culture findings as regards the significance of species, the quantity of growth and, most controversially, the results of in vitro tests on drug susceptibility [10]. For decades, M. gordonae was considered to be nonpathogenic and most likely an indicator of laboratory contamination in positive cultures. With at least one reliable case, we can be relatively certain that this skotochromogenous species can in fact be the causative agent of mycobacterial disease [11]. Nevertheless, the rarity of such cases is still a reason to be cautious on this point. Incidentally, M. terrae is another suspect of this kind. For the clinician, it is the appraisal of newly detected species which may be noticeably more pathogenic than close relatives that is most challenging [12].

To avoid misinterpretations, experienced authors and scientific societies have sought for criteria indicating relevant growth in order to be able to distinguish colonisation, or contamination, from disease. The 1997 statement by the American Thoracic Society (ATS) is still considered to be the major milestone [6]. In nonsterile specimens, these criteria are invariably of a quantitative nature; positive smears are more indicative than negative ones, the more positive cultures the better. However, even with a clear resistance-reducing focus, such as bronchiectasis or post-tuberculous cavities, and histological evidence of granulomata, the indication for treatment is finally based upon the patient's overall condition. Thus, the decision is made by the clinician, who may, in view of the sometimes rather uncomfortable effects the drugs can have, be wise enough to keep under observation even some of those patients who fulfil consensus criteria for mycobacterial disease. Optimal conservative treatment of underlying diseases should not be underestimated, either in this or other contexts, despite the fact that drug treatment has improved over the decades, and patients with bronchiectasis and chronic bronchitis, in particular, should profit from such an approach. Is this a mere opinion? The ATS statement is full of opinions, and rightly so!

It is, therefore, hard to understand why the report on the only prospective randomised study on the treatment of non-HIV patients with $M$. avium-intracellulare, $M$. malmoense and $M$. xenopi disease, which was carried out by the British Thoracic Society (BTS), failed to say anything at all about the treatment of the underlying diseases [13]. This study group found a higher death rate in the group treated with rifampin, ethambutol and isoniazide than in the group treated with rifampin and ethambutol only, although the microbiological response rate was higher with the three-drug regimen. The authors seemed well aware of the methodological difficulties involved in interpreting this outcome. Fortunately, their bold conclusion that the two-drug regimen should be recommended for regular treatment was already outdated at the time of publication, as in the meantime clarithromycin had been shown to be highly effective both in vitro and in vivo.
The BTS conclusions as to the ineffectiveness of in vitro susceptibility tests also appeared questionable. In view of the fact that many more difficulties are associated with the treatment of NTM, these results should not simply be interpreted in a similar manner to the comfortable thresholds used for M. tuberculosis. For example, in a series of M. xenopi strains from the current author's institute, eight out of 15 showed a minimal inhibitory concentration of $0.25 \mu \mathrm{g} \cdot \mathrm{mL}^{-1}$ for isoniazide [11]. A conventional dose of $3-5 \mathrm{mg} \cdot \mathrm{kg}^{-1}$ body weight produces a peak blood concentration of $\sim 5 \mu \mathrm{g} \cdot \mathrm{mL}^{-1}$; the drug penetrates well into all body fluids and cavities, producing concentrations similar to those found in serum [14]. A microbiologist would rate the $0.25 \mu \mathrm{g} \cdot \mathrm{mL}^{-1}$ concentration as only "intermediate" and possibly be wide of the mark. Even more controversially, the majority of $M$. kansasii strains, but not all, show minimal inhibitory concentrations of $1 \mu \mathrm{g} \cdot \mathrm{mL}^{-1}$ for isoniazide, which many microbiologists relentlessly declare "resistant", whereas clinicians feel confident using this drug. For M. avium-intracellulare, in vitro testing shows a greater variability of minimal inhibitory concentrations for potential agents, a finding which may be a guide for alternatives to the so-called standard combinations. Since the results of treatment with recommended regimens that are specific for given species remain unsatisfactory, we might ask ourselves whether we are not falling prey to too narrow an interpretation if we only recommend susceptibility tests in the event of expected, or actual, treatment failure, provided that the clinician is aware of the methodological peculiarities. For uncommon or new species, there is no reason at all to abandon the option of susceptibility tests from the start.

However, leaving aside these uncertainties, where has there been real progress within the last 25 yrs? Rapid and precise recognition of species using fluid cultures and the methods of molecular biology has already become routine. New drugs, such as clarithromycin and moxifloxacin, but not so much rifabutin, should improve treatment results in daily practice $[3,15]$. In future, we may also expect to obtain faster and more differentiated information on the properties of nontuberculous mycobacterial strains with the aid of microarray techniques [16]. Genetic studies of both the host and visitor could finally reveal why only a minority of patients with locally reduced resistance in the lung are affected by ubiquitous mycobacteria $[17,18]$.

As far as treatment is concerned, we might do better to abstain from simple solutions. The gap between evidence and experience can probably only be closed if fixed combinations are compared with an individualised approach based upon the concerted, interdisciplinary judgement of undogmatic partners, as clinicians and microbiologists should always be.

\section{REFERENCES}

1 Shafran SD, Singer J, Zarowny DP, et al. A comparison of two regimens for the treatment of Mycobacterium avium complex bacteremia in AIDS: rifabutin, ethambutol, and clarithromycin versus rifampin, ethambutol, clofazimine, and ciprofloxacin. Canadian HIV Trials Network Protocol 010 Study Group. N Engl J Med 1996; 335: 377-383.

2 May T, Brel F, Beuscart C, et al. Comparison of combination therapy regimens for treatment of human immunodeficiency 
virus-infected patients with disseminated bacteremia due to Mycobacterium avium. ANRS Trial 033 Curavium Group. Agence Nationale de Recherche sur le Sida. Clin Infect Dis 1997; 25: 621-629.

3 Field SK, Cowie RL. Lung disease due to the more common nontuberculous mycobacteria. Chest 2006; 129: 1653-1672.

4 Wolinsky E. Nontuberculous mycobacteria and associated diseases. Am Rev Respir Dis 1979; 119: 107-159.

5 Radenbach KL. Diagnostische und therapeutische Fortschritte bei nichttuberkulösen Mykobakteriosen. [Diagnostic and therapeutic progress in nontuberculous mycobacterioses.]. Prax Klin Pneumol 1985; 39: 43-49.

6 American Thoracic Society. Diagnosis and treatment of disease caused by nontuberculous mycobacteria. Am J Respir Crit Care Med 1997; 156: S1-S25.

7 Subcommittee of the Joint Tuberculosis Committee of the British Thoracic Society. Management of the opportunistic mycobacterial infections, Joint Tuberculosis Committee guidelines 1999. Thorax 2000; 55: 210-218.

8 Dailloux M, Abalain ML, Laurain C, et al. and the French Mycobacteria Study Group. Respiratory infections associated with nontuberculous mycobacteria in non-HIV patients. Eur Respir J 2006; 28: 1211-1215.

9 Fowler SJ, French J, Screaton NJ, et al. Nontuberculous mycobacteria in bronchiectasis: prevalence and patient characteristics. Eur Respir J 2006; 28: 1204-1210.

10 Woods GL. Susceptibility testing for mycobacteria. Clin Infect Dis 2000; 31: 1209-1215.

11 Gößwald A. Klinik, Diagnostik, Therapie und Verlauf pulmonaler Infektionen durch nichttuberkulöse Mykobakterien bei HIV-negativen Patienten in Berlin 1986-1998. Diss. med., Freie Universität, Berlin, 2004.

12 Roth A, Reischl U, Schönfeld N, et al. Mycobacterium heckeshornense sp. nov., a new pathogenic slowly growing
Mycobacterium sp. causing cavitary lung disease in an immunocompetent patient. J Clin Microbiol 2000; 38: 4102-4107.

13 Research Committee of the British Thoracic Society. First randomised trial of treatments for pulmonary disease caused by $M$. avium intracellulare, M. malmoense, and $M$. xenopi in HIV negative patients: rifampicin, ethambutol and isoniazid versus rifampicin and ethambutol. Thorax 2001; 56: 167-172.

14 Bass JB, Farer LS, Hopewell PC, et al. Treatment of tuberculosis and tuberculosis infection in adults and children. American Thoracic Society and The Centers for Disease Control and Prevention. Am J Respir Crit Care Med 1994; 149: 1359-1374.

15 Rodriguez Diaz JC, Lopez M, Ruiz M, Royo G. In vitro activity of new fluoroquinolones and linezolid against nontuberculous mycobacteria. Int J Antimicrob Agents 2003; 21: 585-588.

16 Aragon LM, Navarro F, Heiser V, Garrigo M, Espanol M, Coll P. Rapid detection of specific gene mutations associated with isoniazid or rifampicin resistance in Mycobacterium tuberculosis clinical isolates using nonfluorescent low-density DNA microarrays. J Antimicrob Chemother 2006; 57: 825-831.

17 Doffinger R, Patel SY, Kumararatne DS. Host genetic factors and mycobacterial infections: lessons from single gene disorders affecting innate and adaptive immunity. Microbes Infect 2006; 8: 1141-1450.

18 Oliveira RS, Sircili MP, Oliveira EMD, Balian SC, FerreiraNeto JS, Leão SC. Identification of Mycobacterium avium genotypes with distinctive traits by combination of IS1245based restriction fragment length polymorphism and restriction analysis of hsp65. J Clin Microbiol 2003; 41: $44-49$. 